\title{
3,4-二苯基香豆素衍生物的合成及其促自然杀伤细胞活性研究
}

\author{
陈 华 ${ }^{a}$ 周利凯 ${ }^{a}$ 李 帅 ${ }^{a}$ 姚玉超 ${ }^{a}$ 谷云景 ${ }^{a}$ \\ 李春晓 $b$ 李 娜 $b$ 孟 明*,b 李小六*, $a$ \\ ( ${ }^{a}$ 河北大学化学与环境科学学院 河北省化学生物学重点实验室 保定 071002) \\ ( $b$ 河北大学医学部 保定 071002)
}

\begin{abstract}
摘要 以水杨酸为起始原料, 经 Fridel-Crafts 酰基化反应、微波促进的 Perkin 反应和两步亲核取代反应, 合成了具有氨 基烷氧侧链的 3,4-二苯基香豆素衍生物 1a 1e. 初步生物活性测试表明, 化合物 1a $\sim 1$ 能显著促进自然杀伤细胞杀伤 活性, 具有较强的免疫调节活性.

关键词 香豆素; Perkin 反应; 自然杀伤细胞; 免疫调节活性
\end{abstract}

\section{Synthesis of 3,4-Biphenyl Coumarin Derivatives and Their Promoting Effects on the Killing Activity of Natural Killing Cell}

\author{
Chen, Hua ${ }^{a} \quad{\text { Zhou, } \text { Likai }^{a} \quad \text { Li, Shuai }^{a} \quad \text { Yao, Yuchao }^{a} \quad \text { Gu, Yunjing }}^{a}$ \\ $\mathrm{Li}$, Chunxiao $^{b} \quad \mathrm{Li}, \mathrm{Na}^{b} \quad$ Meng, Ming ${ }^{*, b} \mathrm{Li}, \mathrm{Xiaoliu}^{*, a}$ \\ $\left({ }^{a}\right.$ Key Laboratory of Chemical Biology of Hebei Province, College of Chemistry and Environmental Science, \\ Hebei University, Baoding 071002) \\ ( ${ }^{b}$ Medical School, Hebei University, Baoding 071002)
}

\begin{abstract}
A series of novel 3,4-biphenyl coumarin derivatives containing an amino side chain $\mathbf{1 a} \sim \mathbf{1 e}$ were synthesized using salicylic acid as starting material via Fridel-Crafts acylation, then the microwave-assisted Perkin reaction and double $\mathrm{S}_{\mathrm{N}} 2$ substitution reactions. The preliminary biological evaluation showed that compounds $\mathbf{1 a} \sim \mathbf{1 e}$ had significant promoting effects on the killing activity of natural killer cell, and hold potential as immunomodulating agents.
\end{abstract}

Keywords coumarin; Perkin reaction; natural killer cell; immunomodulating activity

免疫是机体重要的生理保护功能, 许多疾病的发 生、发展与机体免疫功能的失调和缺陷密切相关. 因此, 从调节机体的免疫功能着手调整机体自身功能、防治机 体疾病已经受到广泛重视 ${ }^{[1]}$. 自然杀伤细胞(natural killer cell, NK)是一类具有自发细胞毒活性的淋巴细胞, 与 $\mathrm{T}, \mathrm{B}$ 淋巴细胞共同构筑机体的免疫屏障 ${ }^{[2]}$. NK 细胞既不 需要抗原预先致敏, 也无需抗体参与, 即可自发地直接 杀伤靶细胞, 属于先天性免疫. 它通过细胞毒作用和分 泌细胞因子, 在机体抗肿瘤、抗感染和免疫调节等方面 发挥重要作用 ${ }^{[3]}$. 通过增强 NK 细胞活性, 杀伤肿瘤细
胞已成为肿瘤治疗的一条新途径 ${ }^{[4]}$. 因此, 寻找发现具 有促进 NK 细胞杀伤活性的新型免疫调节剂, 在肿瘤、 病毒性疾病治疗方面具有重要的意义. 虽然生物类制剂 如干扰素、白介素以及植物多糖如香菇多糖、黄芪多糖 等对 NK 杀伤细胞活性有显著的增强作用 ${ }^{[5,6]}$, 但化学合 成的小分子免疫调节剂因其在治疗自身免疫疾病方面 独特的优势, 如良好的化学及酶学稳定性、适应多种剂 型和给药途径的需要, 且成本较低、纯度高、在提高效 价的同时可降低毒副作用等, 成为新型免疫调节剂的研 发热点之一 ${ }^{[7]}$. 然而, 免疫调节的复杂性及靶点不确定

\footnotetext{
*E-mail: lixl@hbu.cn, mengming127@163.com

Received August 21, 2012; revised September 27, 2012; published online October 9, 2012.

Project supported by the National Natural Science Foundation of China (Nos. 20902016, 20972039), the Medicinal Joint Funds of the Natural Science Foundation of Hebei Province and Shijiazhuang Pharmaceutical Group Foundation (No. B2011201169) and the Natural Science Foundations of Education Department of Hebei Province (No. ZH2011110).

国家自然科学基金(Nos. 20902016, 20972039)、河北省自然科学基金石药集团医药联合基金(No. B2011201169)和河北省教育厅自然科学基金(No. ZH2011110)资助项目.
} 
性，致使新型小分子免疫调节剂的设计和发现面临很大 困难和挑战.

从天然产物中发现药物先导依然是现代药物研发 的重要途径之一. 香豆素作为一类具有广谱生物活性的 天然产物, 其衍生物多表现出良好的抗肿瘤、抗病毒和 抗菌等活性 ${ }^{[8,9]}$, 而其在免疫调节方面的活性研究却极 少有报道. 基于三苯乙烯骨架的 3,4-二芳基香豆素衍生 物是一类结构新颖的选择性雌激素调节剂 (SER$\mathrm{Ms})^{[10 \sim 12]}$, 如 SP500263 (图 1), 对雌激素依赖的乳腺癌 (MCF-7)有很强的拮抗作用 ${ }^{[13,14]}$. 最近, 实验室合成了 系列带有氨基烷氧侧链的 3,4-二苯基香豆素衍生物 $(1 \mathbf{a} \sim 1 \mathbf{e}$, 图 1), 但所合成的化合物没有抗 MCF-7 和肺 癌(A549)细胞增殖活性. 鉴于肿瘤的发生、发展与免疫 的密切关联, 我们初步测试了化合物的免疫调节活性. 结果表明，化合物 1a 1e 对免疫 $\mathrm{T}$ 淋巴细胞的增殖作用 不明显, 但却表现出显著的刺激 NK 细胞杀伤活性的作 用，提示该类化合物可能是一类新型的小分子免疫调节 剂. 在此, 本文将主要报道此类 3,4-二苯基香豆素衍生 物的合成及其初步的对 NK 杀伤细胞活性的促进作用.

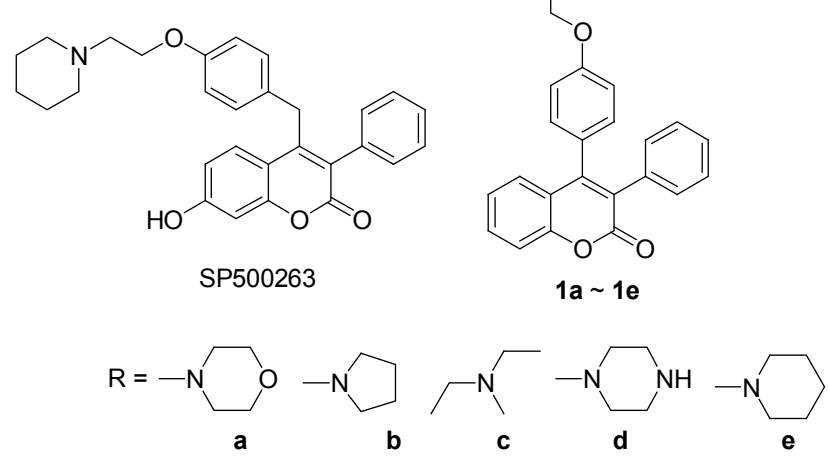

图 1 SP500263 和 3,4-二苯基香豆素衍生物 1a 1e 的结构 Figure 1 Structures of 3,4-biphenyl coumarin derivatives 1a 1e and SP500263

\section{1 结果与讨论}

\section{$1.13,4$-二苯基香豆素衍生物 $1 \mathrm{a} \sim 1 \mathrm{e}$ 的合成}

3,4-二苯基香豆素衍生物 1a 1e 的合成如 Scheme 1 所示. 以水杨酸和苯甲醚为起始原料, 在氯化锌 $\left(\mathrm{ZnCl}_{2}\right)$ 催化下经 Fridel-Crafts 酰基化反应得到 2-羟基4'-甲氧基二苯酮(2). 在此反应过程中, 同时伴有水杨酸 苯酯 $\left(\mathbf{2}^{\prime}\right)$ 的生成. 造成该副产物的原因是苯甲醚在路易 斯酸作用下断裂甲氧键, 生成的苯酚进一步与水杨酸发 生酯化. 随后, 化合物 2 与苯乙酸经 Perkin 反应 ${ }^{[15]}$ 得到 化合物 3. 该反应在微波辐射条件下进行, 反应时间由 传统的加热回流 3 5 h 缩短到 $20 \mathrm{~min}$, 且收率较高. 进 一步, 化合物 3 在三溴化硼 $\left(\mathrm{BBr}_{3}\right)$ 作用下脱除甲基 ${ }^{[16]}$ 得
化合物 4, 接着在碱性条件下，与二溴乙烷发生单取代 反应，得到化合物 $\mathbf{5}$. 化合物 $\mathbf{5}$ 再经与系列胺的亲核取 代反应，合成了具有氨基烷氧侧链的 3,4-二苯基香豆素 衍生物 1a 1e. 该步胺取代反应同样在微波辐射条件 下可以高效的发生，反应时间缩短到 $15 \mathrm{~min}$, 收率在 $64.7 \% \sim 86.4 \%$. 化合物结构经核磁共振 $\left({ }^{1} \mathrm{H}\right.$ 和 ${ }^{13} \mathrm{C}$ NMR)、质谱(ESI-MS)和元素分析等方法确定.
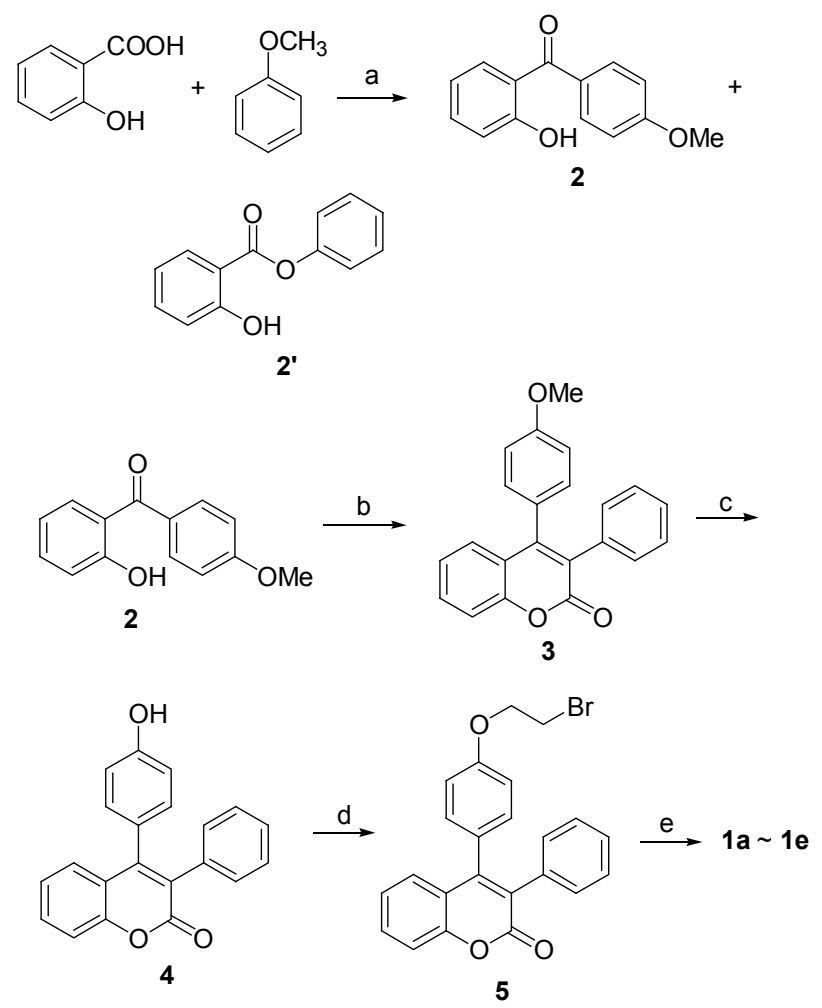

Reagents and conditions: a) $\mathrm{ZnCl}_{2}, \mathrm{POCl}_{3}, 65{ }^{\circ} \mathrm{C}, 2 \mathrm{~h}$; b) Benzene acetic acid, $\mathrm{K}_{2} \mathrm{CO}_{3}, \mathrm{Ac}_{2} \mathrm{O}$, M.W., $130{ }^{\circ} \mathrm{C}, 20 \mathrm{~min}$; c) $\mathrm{BBr}_{3}, \mathrm{CH}_{2} \mathrm{Cl}_{2}, 0$ ${ }^{\circ} \mathrm{C}$; d) $\mathrm{BrCH}_{2} \mathrm{CH}_{2} \mathrm{Br}, \mathrm{K}_{2} \mathrm{CO}_{3}$, Acetone; e) $\mathrm{RNH}$ (3.0 equiv.), $\mathrm{AgNO}_{3}$ (1.0 equiv.), THF, M.W., $125^{\circ} \mathrm{C}, 15 \mathrm{~min}$

\section{Scheme 1}

\section{2 化合物对 NK 杀伤细胞活性的促进作用}

以未处理的脾细胞为空白对照, 临床用免疫增强剂 匹多莫德 (pidotimod) ${ }^{[17]}$ 作用的脾细胞为阳性对照, 测试 了化合物 3, 4 和 $1 \mathrm{a} \sim 1 \mathrm{e}\left(5,50 \mu \mathrm{mol} \cdot \mathrm{L}^{-1}\right)$ 对小鼠 NK 淋巴 细胞杀伤活性的影响. 设不加药的脾细胞为 $100 \%$, 测 试结果如图 1 所示. 结果表明, 含有氨基烷氧侧链的 3,4-二苯基香豆素衍生物 $\mathbf{1} \mathbf{a} \sim \mathbf{1 e}$ 在所测浓度下均能有效 促进 NK 细胞杀伤活性, 杀伤活性率分别提高 $113 \%$, $96 \%, 129 \%, 61 \%, 119 \%\left(50 \mu \mathrm{mol} \cdot \mathrm{L}^{-1}\right)$ 和 $106 \%, 78 \%$, $35 \%, 36 \%, 34 \%\left(5 \mu \mathrm{mol} \cdot \mathrm{L}^{-1}\right)$, 较相同浓度下匹多莫德 $\left(84 \%, 32 \%\right.$ 分别在 50 和 $\left.5 \mu \mathrm{mol} \cdot \mathrm{L}^{-1}\right)$ 的作用要强. 其中, 化合物 $1 \mathrm{a}$ 和 $1 \mathrm{~b}$ 无论在高浓度还是在低浓度下, 都能显 著的促进 NK 细胞杀伤活性. 此外, 化合物 $1 \mathrm{a} \sim 1 \mathrm{e}$ 分别 
单独作用于测试中用到的脾细胞(效应细胞)和 YAC1 (淋巴瘤细胞、靶细胞)时, 均未显示细胞毒性 $\left(\mathrm{IC}_{50}>\right.$ $\left.200 \mu \mathrm{mol} \cdot \mathrm{L}^{-1}\right)$, 提示该类化合物可能是一类新型的高效 低毒的小分子免疫调节剂. 而不含有氨基烷氧侧链的 3,4-二苯基香豆素 3 和 4, 均没有促 NK 细胞杀伤活性, 表明氨基烷氧侧链在此类化合物在对 $\mathrm{NK}$ 细胞的作用上 有着重要的影响. 进一步的合成及活性研究正在进行.

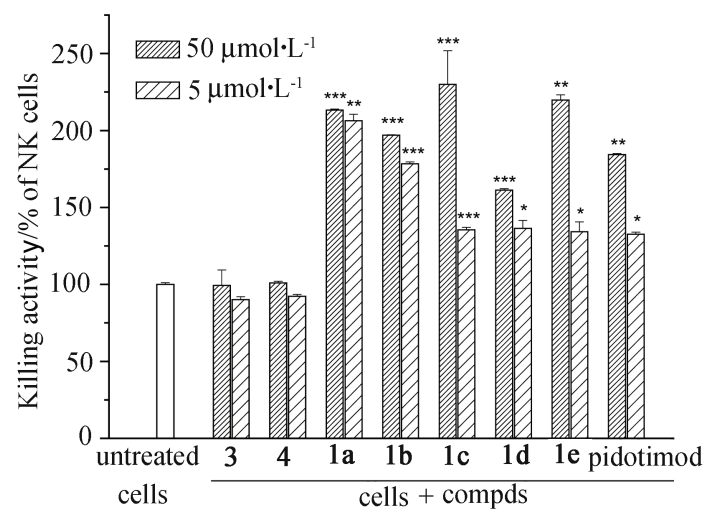

图 1 化合物 3, 4, 1a 1e 和匹多莫德对小鼠 NK 淋巴细胞杀 伤活性的影响

Figure 1 The effects of compounds 3, 4, 1a $\sim 1$ e and pidotimod on NK cells killing activity

The concentrations of the tested compounds were 50 and $5 \mu \mathrm{mol} \cdot \mathrm{L}^{-1}$, respectively. Data are means \pm SEM of at least three independent experiments. ${ }^{*} p<0.05,{ }^{* *} p<0.01,{ }^{* * *} p<0.001$ with respect to the untreated NK cells

\section{2 结论}

综上所述, 本文以水杨酸为起始原料合成了具有氨 基烷氧侧链的 3,4-二苯基香豆素衍生物 1a 1e. 化合物 1a 1e 能显著促进 NK 细胞杀伤活性, 且氨基烷氧侧链 的存在对活性影响较大. 相同浓度下, 化合物 $\mathbf{1 a} \sim 1 \mathrm{e}$ 的 促 NK 细胞杀伤活性较阳性对照匹多莫德强, 提示该类 化合物可能是一类新型的小分子免疫调节剂.

\section{3 实验部分}

\section{1 仪器与试剂}

微波合成仪为美国 CEM 公司的 DISCOVER SClass; 熔点由 $\mathrm{SGW}^{\circledR} \mathrm{X}-4$ 显微熔点仪(温度计未校正)测 定; 核磁共振谱用 BRUKER AC-P600 (600 MHz)型核磁 共振仪测定, TMS 为内标; 质谱(ESI)用 Agilent G6300 离子阱液相质谱联用仪; 元素分析用 HEKAEUS (CHNO, rapid)型元素分析仪; 细胞光密度用 BioRad 3550 型酶标仪测定; 层析用硅胶(200 300 目)为青岛海 洋化工厂产品. 本文所用其他试剂均为分析纯, 无水试 剂均按常规方法处理, 水为二次蒸馏水.

\section{2 实验方法}

3.2 .1 (2-羟基苯基)(4-甲氧基苯基)甲基酮(2)和 2-羟 基苯甲酸苯酯 $\left(2^{\prime}\right)$ 的合成

$50 \mathrm{~mL}$ 圆底烧瓶中加入 $414 \mathrm{mg}(3.0 \mathrm{mmol})$ 水杨酸, $1.02 \mathrm{~g}(7.5 \mathrm{mmol})$ 无水氯化锌, $7 \mathrm{~mL}$ 三氯氧磷, 室温搅拌 $20 \mathrm{~min}$, 加入 $326 \mu \mathrm{L}(3.0 \mathrm{mmol})$ 苯甲醚, 保持油浴温度 $60 \sim 65{ }^{\circ} \mathrm{C}$, 反应 $2 \mathrm{~h}$, 将反应液倒入 $10 \mathrm{~mL}$ 冰水中搅拌, 乙酸乙酯 $(10 \mathrm{~mL} \times 2)$ 萃取, 有机相依次用饱和碳酸氢钠 溶液洗去有机相中的酸性物质, 水洗, 饱和食盐水洗, 无水硫酸镁 $\left(\mathrm{MgSO}_{4}\right)$ 干燥. 抽滤后旋转蒸发除去溶剂, 硅胶柱层析 $[V($ 石油醚 $): V($ 乙酸乙酯 $)=60 ： 1]$ 分离得 $340 \mathrm{mg}$ 化合物 2 和 $140 \mathrm{mg}$ 化合物 $\mathbf{2}^{\prime}$ ，化合物表征数据 与文献[18]报道值一致.

化合物 2: 黄色油状物, 产率 50.0\%. ${ }^{1} \mathrm{H}$ NMR (600 $\left.\mathrm{MHz}, \mathrm{CDCl}_{3}\right) \delta: 3.88\left(\mathrm{~s}, 3 \mathrm{H}, \mathrm{OCH}_{3}\right), 6.88(\mathrm{t}, J=7.8 \mathrm{~Hz}$, 1H, ArH), 6.98 (d, $J=9.0 \mathrm{~Hz}, 2 \mathrm{H}, \operatorname{ArH}), 7.05$ (d, $J=8.4$ $\mathrm{Hz}, 2 \mathrm{H}, \mathrm{ArH}), 7.48$ (t, J=7.2 Hz, 1H, ArH), 7.62 (d, $J=$ $7.8 \mathrm{~Hz}, 1 \mathrm{H}, \mathrm{ArH}), 7.70$ (d, J=8.4 Hz, 2H, ArH), 11.98 (s, $1 \mathrm{H}, \mathrm{OH}) ; \mathrm{MS}(\mathrm{ESI}) \mathrm{m} / z: 251.1\left([\mathrm{M}+\mathrm{Na}]^{+}\right)$.

化合物 2': 油状物, 产率 21.8\%. ${ }^{1} \mathrm{H}$ NMR (600 $\left.\mathrm{MHz}, \mathrm{CDCl}_{3}\right) \delta: 6.85$ (td, $\left.J=7.8,0.6 \mathrm{~Hz}, 1 \mathrm{H}, \mathrm{ArH}\right), 6.92$ (dd, $J=8.4,0.6 \mathrm{~Hz}, 1 \mathrm{H}, \mathrm{ArH}), 7.09$ (dd, $J=8.4,0.6 \mathrm{~Hz}$, 2H, ArH), 7.19 (d, $J=7.8 \mathrm{~Hz}, 1 \mathrm{H}, \mathrm{ArH}), 7.33$ (t, $J=7.8$ $\mathrm{Hz}, 2 \mathrm{H}, \mathrm{ArH}), 7.41$ (td, $J=8.4,1.2 \mathrm{~Hz}, 1 \mathrm{H}, \mathrm{ArH}), 7.95$ (dd, $J=7.8,1.2 \mathrm{~Hz}, 1 \mathrm{H}, \mathrm{ArH}), 10.41$ (d, $J=1.2 \mathrm{~Hz}, 1 \mathrm{H}$, $\mathrm{OH})$; MS (ESI) $m / z: 237.0\left([\mathrm{M}+\mathrm{Na}]^{+}\right)$.

3.2.2 4-(4-甲氧基苯基)-3-苯基苯并吡喃-2-酮(3)的 合成

$10 \mathrm{~mL}$ 的微波管中加入 $348 \mathrm{mg}(1.5 \mathrm{mmol})$ 化合物 2, $414 \mathrm{mg}(3.0 \mathrm{mmol})$ 碳酸钾, $249 \mathrm{mg}(1.2 \mathrm{mmol})$ 苯乙酸和 $600 \mu \mathrm{L}$ 乙酸酐. 微波 $130{ }^{\circ} \mathrm{C}$ 反应 $20 \mathrm{~min}$. 加水淬灭反应, 乙酸乙酯 $(10 \mathrm{~mL} \times 2)$ 萃取, 收集有机相, 饱和食盐水水 洗, 无水硫酸镁干燥. 抽滤, 减压除去溶剂, 硅胶柱层 析 $[V$ (石油醚)： $V$ (乙酸乙酯 $)=3 ： 1$ ]分离得 $320 \mathrm{mg}$ 化 合物 3. 白色固体, 产率 $64.0 \%$, m.p. 222.6 223.8 ${ }^{\circ} \mathrm{C}$; ${ }^{1} \mathrm{H}$ NMR $\left(600 \mathrm{MHz}, \mathrm{CDCl}_{3}\right) \delta: 3.79\left(\mathrm{~s}, 3 \mathrm{H}, \mathrm{OCH}_{3}\right), 6.82$ (d, $J=9.0 \mathrm{~Hz}, 2 \mathrm{H}, \mathrm{ArH}), 7.03$ (d, $J=9.0 \mathrm{~Hz}, 2 \mathrm{H}, \mathrm{ArH})$, $7.13(\mathrm{dd}, J=7.8,1.2 \mathrm{~Hz}, 2 \mathrm{H}, \mathrm{ArH}), 7.17 \sim 7.20(\mathrm{~m}, 4 \mathrm{H}$, ArH), 7.29 (dd, $J=7.8,1.2 \mathrm{~Hz}, 1 \mathrm{H}, \mathrm{ArH}), 7.42$ (dd, $J=$ 8.4, $1.2 \mathrm{~Hz}, 1 \mathrm{H}, \mathrm{ArH}), 7.53$ (td, $J=8.4,1.8 \mathrm{~Hz}, 1 \mathrm{H}, \mathrm{ArH})$; MS (ESI) $m / z: 329.2\left([\mathrm{M}+\mathrm{H}]^{+}\right), 351.1\left([\mathrm{M}+\mathrm{Na}]^{+}\right)$. Anal. calcd for $\mathrm{C}_{22} \mathrm{H}_{16} \mathrm{O}_{3}$ : C 80.47, $\mathrm{H} 4.91$; found $\mathrm{C} 80.36, \mathrm{H}$ 4.85 . 


\subsubsection{4-(4-差基苯基)-3-苯基苯并吡喃-2-酮(4)的合} 成

$25 \mathrm{~mL}$ 圆底烧瓶中加入 $328 \mathrm{mg}(1.0 \mathrm{mmol})$ 化合物 $\mathbf{3}$, $4 \mathrm{~mL}$ 新蒸二氯甲烷, 冰浴搅拌下滴加 $4 \mathrm{~mL}$ 浓度为 1.0 $\mathrm{mol} / \mathrm{L}$ 的三溴化硼的二氯甲烷溶液. TLC 监测反应完成, 加水淬灭. 反应液用乙酸乙酯 $(15 \mathrm{~mL} \times 2)$ 萃取, 收集有 机相, 饱和食盐水洗, 无水硫酸镁干燥. 抽滤, 减压除 去溶剂得 $300 \mathrm{mg}$ 化合物 4. 白色固体, 产率 95.5\%. m.p. $295.0 \sim 297.0{ }^{\circ} \mathrm{C} ;{ }^{1} \mathrm{H}$ NMR (600 MHz, CD 3 OD) $\delta: 6.72$ (d, $J=8.4 \mathrm{~Hz}, 2 \mathrm{H}, \mathrm{ArH}), 6.97$ (d, $J=8.4 \mathrm{~Hz}, 2 \mathrm{H}, \operatorname{ArH}), 7.13$ (d, $J=7.2 \mathrm{~Hz}, 2 \mathrm{H}, \mathrm{ArH}), 7.18 \sim 7.21(\mathrm{~m}, 3 \mathrm{H}, \mathrm{ArH}), 7.28$ (t, $J=7.8 \mathrm{~Hz}, 1 \mathrm{H}, \mathrm{ArH}), 7.33$ (d, $J=7.8 \mathrm{~Hz}, 1 \mathrm{H}, \mathrm{ArH})$, $7.44(\mathrm{~d}, J=8.4 \mathrm{~Hz}, 1 \mathrm{H}, \mathrm{ArH}), 7.61(\mathrm{td}, J=8.4,1.2 \mathrm{~Hz}, 1 \mathrm{H}$, ArH); MS (ESI) $m / z: 337.1\left([\mathrm{M}+\mathrm{Na}]^{+}\right)$. Anal. calcd for $\mathrm{C}_{21} \mathrm{H}_{14} \mathrm{O}_{3}$ : C 80.24, $\mathrm{H} 4.49$; found $\mathrm{C} 80.18, \mathrm{H} 4.60$.

3.2 .4 4-[4-(2-溴乙氧基)苯基]-3-苯基苯并吡喃-2-酮 (5) 的合成

$50 \mathrm{~mL}$ 圆底烧瓶中加入 $314 \mathrm{mg}(1.0 \mathrm{mmol})$ 化合物 4, $400 \mu \mathrm{L}(5.0 \mathrm{mmol}) 1,2$ 二二溴乙烷, $553 \mathrm{mg}$ (4.0 mmol)碳酸 钾, $10 \mathrm{~mL}$ 无水丙酮, 加热回流至 TLC 监测反应完成. 减压除去溶剂, 硅胶柱层析 $[V$ (石油醚) $: V$ ( 乙酸乙酯 $)=$ $3: 1$ ] 分离得 $252 \mathrm{mg}$ 化合物 5 . 白色固体, 产率 $60.0 \%$. m.p. $176.8 \sim 177.4{ }^{\circ} \mathrm{C} ;{ }^{1} \mathrm{H}$ NMR $\left(600 \mathrm{MHz}, \mathrm{CDCl}_{3}\right) \delta: 3.63$ (t, $\left.J=6.0 \mathrm{~Hz}, 2 \mathrm{H}, \mathrm{CH}_{2}\right), 4.27\left(\mathrm{t}, J=6.0 \mathrm{~Hz}, 2 \mathrm{H}, \mathrm{OCH}_{2}\right.$ ), $6.84(\mathrm{~d}, J=8.4 \mathrm{~Hz}, 2 \mathrm{H}, \operatorname{ArH}), 7.05$ (d, $J=8.4 \mathrm{~Hz}, 2 \mathrm{H}$, $\mathrm{ArH}), 7.12 \sim 7.14(\mathrm{~m}, 2 \mathrm{H}, \mathrm{ArH}), 7.17 \sim 7.23(\mathrm{~m}, 4 \mathrm{H}, \mathrm{ArH})$, $7.27(\mathrm{dd}, J=8.4,1.8 \mathrm{~Hz}, 2 \mathrm{H}, \operatorname{ArH}), 7.42$ (d, $J=7.8 \mathrm{~Hz}$, $2 \mathrm{H}, \mathrm{ArH}), 7.53$ (td, $J=8.4,1.2 \mathrm{~Hz}, 1 \mathrm{H}, \mathrm{ArH})$; MS (ESI) $m / z: 443.3\left([\mathrm{M}+\mathrm{Na}]^{+}\right)$. Anal. calcd for $\mathrm{C}_{23} \mathrm{H}_{17} \mathrm{BrO}_{3}: \mathrm{C}$ 65.57, H 4.07; found C 65.42, H 4.01.

\section{2 .5 化合物 $1 \mathrm{a} \sim 1 \mathrm{e}$ 的合成}

以化合物 $1 \mathrm{c}$ 的合成为例: $10 \mathrm{~mL}$ 微波管中加入化合 物 $84 \mathrm{mg}(0.2 \mathrm{mmol}) \mathbf{4 a}, 62 \mu \mathrm{L}(0.6 \mathrm{mmol})$ 二乙胺, $34 \mathrm{mg}$ (0.2 mmol) 硝酸银, $2 \mathrm{~mL}$ 四氢呋喃, 微波 $125{ }^{\circ} \mathrm{C}$ 加热 15 min. 将反应液转移至 $50 \mathrm{~mL}$ 的圆底烧瓶中, 干法上样, 硅胶柱层析 $[V$ (石油醚) : $V$ (乙酸乙酯 $): V$ (三乙胺 $)=$ $75: 25: 2]$ 分离得 $53 \mathrm{mg}$ 化合物 1c.

用同样的方法可以得到 $64 \mathrm{mg} \mathrm{1a}, 55 \mathrm{mg} \mathrm{1b}, 72 \mathrm{mg}$ 1e, $74 \mathrm{mg} \mathbf{1 d}$.

4-[4-(2-吗啉乙氧基)苯基]-3-苯基苯并吡喃-2-酮 (1a): 白色固体, 产率 $74.9 \%$. m.p. $146.3 \sim 148.1{ }^{\circ} \mathrm{C} ;{ }^{1} \mathrm{H}$ NMR (600 MHz, $\mathrm{CDCl}_{3}$ ) $\delta: 2.57$ (brs, 4H, $\left.\mathrm{CH}_{2}\right), 2.79$ (t, $\left.J=6.0 \mathrm{~Hz}, 2 \mathrm{H}, \mathrm{CH}_{2}\right), 3.73\left(\mathrm{t}, J=4.8 \mathrm{~Hz}, 4 \mathrm{H}, \mathrm{CH}_{2}\right), 4.08(\mathrm{t}$, $\left.J=6.0 \mathrm{~Hz}, 2 \mathrm{H}, \mathrm{OCH}_{2}\right), 6.82(\mathrm{~d}, J=9.0 \mathrm{~Hz}, 2 \mathrm{H}, \mathrm{ArH}), 7.02$ $(\mathrm{d}, J=9.0 \mathrm{~Hz}, 2 \mathrm{H}, \mathrm{ArH}), 7.12 \sim 7.13(\mathrm{~m}, 2 \mathrm{H}, \mathrm{ArH}), 7.16 \sim$ $7.22(\mathrm{~m}, 4 \mathrm{H}, \mathrm{ArH}), 7.27$ (dd, $J=8.4,1.2 \mathrm{~Hz}, 1 \mathrm{H}, \mathrm{ArH})$, $7.41(\mathrm{dd}, J=8.4,0.6 \mathrm{~Hz}, 1 \mathrm{H}, \mathrm{ArH}), 7.53(\mathrm{td}, J=9.0,1.8$ $\mathrm{Hz}, 1 \mathrm{H}, \mathrm{ArH}) ;{ }^{13} \mathrm{C} \mathrm{NMR}\left(\mathrm{CDCl}_{3}, 150 \mathrm{MHz}\right) \delta: 54.1(2 \mathrm{C})$, 57.3, 65.8, 66.7, 66.9, 114.4 (2C), 120.3, 121.5, 123.8, $126.6,127.1,127.3,127.8,128.4,128.8(2 \mathrm{C}), 130.7$ (2C), 130.8 (2C), 134.3, 151.5, 154.9, 158.6, 161.6; MS (ESI) $m / z: 428.2\left([\mathrm{M}+\mathrm{H}]^{+}\right), 450.2\left([\mathrm{M}+\mathrm{Na}]^{+}\right)$. Anal. calcd for $\mathrm{C}_{27} \mathrm{H}_{25} \mathrm{NO}_{4}$ : C 75.86, H 5.89, N 3.28; found $\mathrm{C} 75.77, \mathrm{H}$ $6.03, \mathrm{~N} 3.26$.

4-\{4-[2-(吡咯烷-1-基)乙氧基]苯基\}-3-苯基苯并吡 喃-2-酮(1b): 白色固体，产率 67.0\%. m.p. 139.7 140.9 ${ }^{\circ} \mathrm{C},{ }^{1} \mathrm{H}$ NMR $\left(600 \mathrm{MHz}, \mathrm{CDCl}_{3}\right) \delta: 1.80 \sim 1.83(\mathrm{~m}, 4 \mathrm{H}$, $\mathrm{CH}_{2}$ ), 2.63 (brs, 4H, $\mathrm{CH}_{2}$ ), 2.90 (t, $J=6.0 \mathrm{~Hz}, 2 \mathrm{H}, \mathrm{CH}_{2}$ ), $4.08\left(\mathrm{t}, J=6.0 \mathrm{~Hz}, 2 \mathrm{H}, \mathrm{OCH}_{2}\right), 6.83(\mathrm{~d}, J=8.4 \mathrm{~Hz}, 2 \mathrm{H}$, $\operatorname{ArH}), 7.01$ (d, $J=8.4 \mathrm{~Hz}, 2 \mathrm{H}, \operatorname{ArH}), 7.12 \sim 7.13(\mathrm{~m}, 2 \mathrm{H}$, ArH), $7.16 \sim 7.23(\mathrm{~m}, 4 \mathrm{H}, \mathrm{ArH}), 7.29(\mathrm{dd}, J=7.8,1.2 \mathrm{~Hz}$, $1 \mathrm{H}, \mathrm{ArH}), 7.41$ (d, $J=8.4 \mathrm{~Hz}, 1 \mathrm{H}, \mathrm{ArH}), 7.52$ (td, $J=8.4$, $1.2 \mathrm{~Hz}, 1 \mathrm{H}, \mathrm{ArH}) ;{ }^{13} \mathrm{C} \mathrm{NMR}\left(\mathrm{CDCl}_{3}, 150 \mathrm{MHz}\right) \delta: 23.5$ (2C), 54.6 (2C), 54.8, 66.7, 113.3 (2C), 121.6, 122.7, 123.6, 125.9 (2C), 126.2, 126.7 (2C), 127.8 (2C), 129.7 (4C), 133.4, 150.6, 153.8, 157.6, 160.7; MS (ESI) $m / z$ : $412.3\left([\mathrm{M}+\mathrm{H}]^{+}\right), 434.2\left([\mathrm{M}+\mathrm{Na}]^{+}\right)$. Anal. calcd for $\mathrm{C}_{27} \mathrm{H}_{25} \mathrm{NO}_{3}$ : C 78.81, H 6.12, N 3.40; found $\mathrm{C} 78.95, \mathrm{H}$ $6.22, \mathrm{~N} 3.35$.

4-\{4-[2-(二乙基胺基)乙氧基]苯基\}-3-苯基苯并吡 喃-2-酮(1c): 白色固体，产率 64.7\%. m.p. 88.3 89.1 ${ }^{\circ} \mathrm{C} ;{ }^{1} \mathrm{H}$ NMR $\left(600 \mathrm{MHz}, \mathrm{CDCl}_{3}\right) \delta: 1.08(\mathrm{t}, J=7.2 \mathrm{~Hz}, 6 \mathrm{H}$, $\mathrm{CH}_{3}$ ), 2.64 (q, $J=7.2 \mathrm{~Hz}, 4 \mathrm{H}, \mathrm{CH}_{2}$ ), 2.88 (t, $J=6.0 \mathrm{~Hz}$, $\left.2 \mathrm{H}, \mathrm{CH}_{2}\right), 4.04\left(\mathrm{t}, J=6.0 \mathrm{~Hz}, 2 \mathrm{H}, \mathrm{OCH}_{2}\right), 6.82$ (d, $J=8.4$ $\mathrm{Hz}, 2 \mathrm{H}, \mathrm{ArH}), 7.01$ (d, J=9.0 Hz, 2H, ArH), 7.12 7.13 (m, 2H, ArH), 7.16 $7.21(\mathrm{~m}, 4 \mathrm{H}, \mathrm{ArH}), 7.28(\mathrm{dd}, J=8.4$, $1.8 \mathrm{~Hz}, 1 \mathrm{H}, \mathrm{ArH}), 7.41$ (d, $J=8.4 \mathrm{~Hz}, 1 \mathrm{H}, \mathrm{ArH}), 7.52$ (td, $J=8.4,1.2 \mathrm{~Hz}, 1 \mathrm{H}, \mathrm{ArH}) ;{ }^{13} \mathrm{C} \mathrm{NMR}\left(\mathrm{CDCl}_{3}, 150 \mathrm{MHz}\right) \delta$ : 11.4, 11.6, 47.7 (2C), 51.4, 66.2, 114.3 (2C), 121.3, 122.4, 123.6, 126.9 (2C), 127.2, 127.7 (2C), 128.8 (2C), 130.8 (4C), 134.4, 151.6, 154.8, 158.6, 161.6; MS (ESI) $\mathrm{m} / \mathrm{z}$ : $412.2\left([\mathrm{M}-\mathrm{H}]^{+}\right)$. Anal. calcd for $\mathrm{C}_{27} \mathrm{H}_{27} \mathrm{NO}_{3}: \mathrm{C} 78.42, \mathrm{H}$ 6.58, N 3.39; found C 78.46, H 6.49, N 3.37.

4-\{4-[2-(哌嗪-1-基)乙氧基]苯基\}-3-苯基苯并吡喃2-酩(1d): 白色固体，产率 86.4\%. m.p. 47.5 48.8 ${ }^{\circ} \mathrm{C}$; ${ }^{1} \mathrm{H}$ NMR (600 MHz, CD $\left.\mathrm{OD}\right) \delta: 2.59$ (brs, 4H, $\mathrm{CH}_{2}$ ), 2.77 $\left(\mathrm{t}, J=5.4 \mathrm{~Hz}, 2 \mathrm{H}, \mathrm{CH}_{2}\right), 2.90 \sim 2.91\left(\mathrm{~m}, 4 \mathrm{H}, \mathrm{CH}_{2}\right), 4.07(\mathrm{t}$, $\left.J=5.4 \mathrm{~Hz}, 2 \mathrm{H}, \mathrm{OCH}_{2}\right), 6.85$ (d, $\left.J=8.4 \mathrm{~Hz}, 2 \mathrm{H}, \mathrm{ArH}\right), 7.02$ (d, $J=9.0 \mathrm{~Hz}, 2 \mathrm{H}, \mathrm{ArH}), 7.09 \sim 7.10$ (m, 2H, ArH), 7.14 $7.15(\mathrm{~m}, 3 \mathrm{H}, \mathrm{ArH}), 7.19 \sim 7.24(\mathrm{~m}, 2 \mathrm{H}, \mathrm{ArH}), 7.37$ (d, $J=$ 
$8.4 \mathrm{~Hz}, 1 \mathrm{H}, \mathrm{ArH}), 7.55$ (td, $J=8.4,1.8 \mathrm{~Hz}, 1 \mathrm{H}, \mathrm{ArH}) ;{ }^{13} \mathrm{C}$ NMR $\left(\mathrm{CDCl}_{3}, 150 \mathrm{MHz}\right) \delta: 44.8,44.9,55.2$ (2C), 55.4, 68.5, 114.3 (2C), 121.8, 123.1, 123.5, 127.1 (2C), 127.3, 127.9 (2C), 129.1 (2C), 130.7 (4C), 134.6, 151.1, 154.6, 158.6, 161.7; MS (ESI) $m / z: 427.3\left([\mathrm{M}+\mathrm{H}]^{+}\right)$. Anal. calcd for $\mathrm{C}_{27} \mathrm{H}_{26} \mathrm{~N}_{2} \mathrm{O}_{3}$ : C 76.03, H 6.14, N 6.57; found C 75.92, H 6.09, N 6.38.

4-\{4-[2-(哌啶-1-基)乙氧基]苯基\}-3-苯基苯并吡喃2-酮(1e): 白色固体，产率 84.6\%. m.p. $144.5 \sim 145.9{ }^{\circ} \mathrm{C}$; ${ }^{1} \mathrm{H}$ NMR (600 MHz, $\mathrm{CDCl}_{3}$ ) $\delta: 1.45$ (brs, 2H, $\mathrm{CH}_{2}$ ), $1.62 \sim$ $1.68\left(\mathrm{~m}, 4 \mathrm{H}, \mathrm{CH}_{2}\right), 2.54$ (brs, 4H, $\left.\mathrm{CH}_{2}\right), 2.79$ (t, $J=6.0 \mathrm{~Hz}$, $\left.2 \mathrm{H}, \mathrm{CH}_{2}\right), 4.09$ (t, $\left.J=6.0 \mathrm{~Hz}, 2 \mathrm{H}, \mathrm{OCH}_{2}\right), 6.81$ (d, $J=8.4$ $\mathrm{Hz}, 2 \mathrm{H}, \mathrm{ArH}), 7.00$ (d, $J=8.4 \mathrm{~Hz}, 2 \mathrm{H}, \mathrm{ArH}), 7.11 \sim 7.12$ (m, 2H, ArH), 7.16 $7.21(\mathrm{~m}, 4 \mathrm{H}, \mathrm{ArH}), 7.28(\mathrm{dd}, J=7.8$, $1.2 \mathrm{~Hz}, 1 \mathrm{H}, \mathrm{ArH}), 7.40$ (d, $J=8.4 \mathrm{~Hz}, 1 \mathrm{H}, \mathrm{ArH}), 7.51(\mathrm{td}$, $J=8.4,1.2 \mathrm{~Hz}, 1 \mathrm{H}, \mathrm{ArH}) ;{ }^{13} \mathrm{C} \mathrm{NMR}\left(\mathrm{CDCl}_{3}, 150 \mathrm{MHz}\right) \delta$ : 23.1, 24.8 (2C), 54.0 (2C), 56.5, 65.5, 114.3 (2C), 122.1, 122.6, 123.7, 127.0 (2C), 127.3, 127.7 (2C), 128.9 (2C), 130.8 (4C), 134.4, 151.6, 154.8, 158.6, 161.6; MS (ESI) $m / z: 426.3\left([\mathrm{M}+\mathrm{H}]^{+}\right)$. Anal. calcd for $\mathrm{C}_{28} \mathrm{H}_{27} \mathrm{NO}_{3}: \mathrm{C}$ 79.03, H 6.40, N 3.29; found C 78.99, H 6.28, N 3.31.

3.2.6 化合物对小鼠 NK 细胞杀伤活性的影响

小鼠脾淋巴细胞悬液的制备：颈椎脱臼处死 $\mathrm{BALb} / \mathrm{C}$ 小鼠，用碘伏浸泡 $5 \mathrm{~min}$, 再用 $75 \%$ 乙醇浸泡小 鼠 $3 \mathrm{~min}$, 沿腹腔中线剪开小鼠腹腔, 取出脾脏置于培 养皿中, 剪去脂肪和筋膜组织, 用 RPMI-1640 培养液漂 洗 $^{[19]}$. 置组织于研磨器中加入适量磷酸盐缓冲液(PBS 液)研磨, 制成脾细胞悬液, 过 200 目笁网, 将得到的细 胞悬液置于离心管中 $1500 \mathrm{r} / \mathrm{min}$ 离心 $15 \mathrm{~min}$. 弃上层清 液, 加入 Tris- $\mathrm{NH}_{4} \mathrm{Cl}$ 溶液 9 倍体积 $0.83 \mathrm{~g} / 100 \mathrm{~mL}$ 的氯 化铵 $\left(\mathrm{NH}_{4} \mathrm{Cl}\right)$ 溶液与 1 倍体积 $2.06 \mathrm{~g} / 100 \mathrm{~mL}$ 的 Tris- $\mathrm{HCl}$ 溶液 $\left(\mathrm{pH}\right.$ 7.65)混合, 用 $\mathrm{NH}_{4} \mathrm{Cl}$ 调节 $\mathrm{pH}$ 值至 7.2) 裂解红 细胞, 室温静置 $5 \mathrm{~min}$, 以除去红细胞, 见离心后所得为 白色沉淀上方只有一层红色膜为最佳状态, 用 RPMI1640 培养液 $1000 \mathrm{r} / \mathrm{min}$ 离心 $10 \mathrm{~min}$ 洗涤细胞 3 次, 以除 去剩余的氯化铵. 将上述收集到的脾细胞置于培养瓶 中, 在 $37{ }^{\circ} \mathrm{C}, 5 \% \mathrm{CO}_{2}$ 培养箱培养 $2 \mathrm{~h}$, 以除去贴壁细胞, 收集未贴壁的细胞悬液即为脾淋巴细胞. 台盼蓝染色计 数, 接种培养, 活细胞数大于 $95 \%$, 备用.

化合物对小鼠 NK 细胞杀伤活性的影响：用四甲基 唑蓝(MTT)法测定 NK 细胞杀伤活性. 以上述方法制备 的小鼠脾淋巴细胞为效应细胞, $\mathrm{YAC}-1$ (淋巴瘤细胞)为 靶细胞, 效应细胞与靶细胞的比例 $(\mathrm{E} / \mathrm{T})$ 为 $50: 1$, 测试 效应细胞对靶细胞的杀伤活性. 将上述小鼠脾淋巴细胞
和 YAC-1 细胞悬浮于含有 $5 \%$ 小牛血清, $100 \mathrm{IU} / \mathrm{mL}$ 盘尼 西林和 $100 \mu \mathrm{g} / \mathrm{mL}$ 链霉素的 RPMI-1640 培养液中培养, 调整细胞浓度分别为 $5 \times 10^{6}$ 个 $/ \mathrm{mL}$ 和 $1 \times 10^{5}$ 个 $/ \mathrm{mL}$. 在 96 孔细胞培养板中, 加入 $90 \mu \mathrm{L} /$ 孔脾淋巴细胞悬液和 YAC-1 细胞悬液 (混合孔, M). 此外, 设置单独的效应细 胞孔( $\mathrm{E}, 90 \mu \mathrm{L}, 5 \times 10^{6}$ 个 $\left./ \mathrm{mL}\right)$ 和靶细胞孔 $(\mathrm{T}, 90 \mu \mathrm{L}, 1 \times$ $10^{5}$ 个 $/ \mathrm{mL}$ ) 为对照组. 在混合孔和效应细胞孔中分别加 入待测化合物，使其终浓度为 5 和 $50 \mu \mathrm{mol} \cdot \mathrm{L}^{-1}$. 所有的 试验均至少重复 3 次，置于 $37{ }^{\circ} \mathrm{C}$, 含 $5 \%$ 的 $\mathrm{CO}_{2}$ 条件下 分别培养 $4 \mathrm{~h}$ 后每孔加 MTT $10 \mu \mathrm{L}(5 \mathrm{~g} / \mathrm{L})$ 继续孵育 $4 \mathrm{~h}$, 弃去上清, 然后每孔加入 $100 \mu \mathrm{L} \mathrm{DMSO}$ 溶解 MTT 甲穊 沉淀, 用微型振荡器振荡混匀, 变色后用酶联免疫检测 仪分别测定 $570 \mathrm{~nm}$ 波长处的吸光度 $A_{570 \mathrm{~nm}}$. 杀伤率计算 $(\%)=\left(A_{\mathrm{T}}+A_{\mathrm{E}}-A_{\mathrm{M}}\right) / A_{\mathrm{T}} \times 100 \%$.

\section{References}

[1] Stockinger, B. Nature 2009, 458, 44.

[2] Sun, J. C.; Lanier, L. L. Nat. Rev. Immunol. 2011, 11, 645.

[3] Thielens, A.; Vivier, E.; Romagné, F. Curr. Opin. Immunol. 2012, $24,239$.

[4] Suck, G. Semin. Cancer Biol. 2006, 16, 412.

[5] Failli, A.; Caggiano, T. J. Curr. Opin. Ther. Pat. 1992, 2, 882.

[6] Zhang, C.; Zhang, J.; Niu, J.; Zhou, Z.; Zhang, J.; Tian, Z. Cytokine 2008, $42,128$.

[7] Werner, G. H.; Jollès, P. Eur. J. Biochem. 1996, 242, 1.

[8] Zhao, H. P.; Donnelly, A. C.; Kusuma, B. R.; Brandt, G. E. L.; Brown, D.; Rajewski, R. A.; Vielhauer, G.; Holzbeierlein, J.; Cohen, M. S.; Blagg, S. J. J. Med. Chem. 2011, 54, 3839.

[9] Stefanachi, A.; Favia, A. D.; Nicolotti, O.; Leonetti, F.; Pisani, L.; Catto, M.; Zimmer, C.; Hartmann, R. W.; Carotti, A. J. Med. Chem. 2011, 54, 1613.

[10] Katzenellenbogen, B. S.; Katzenellebogen, J. K. Science 2002, 295, 2380.

[11] Jordan, V. C. J. Med. Chem. 2003, 46, 883.

[12] Jordan, V. C. J. Med. Chem. 2003, 46, 1081.

[13] Brady, H.; Desai, I.; Gayo-Fung, L. M.; Khammungkhune, S.; McKie, J. A.; O'Leary, E.; Pascasio, L.; Sutherland, M. K.; Anderson, D. W.; Bhagwat, S. S.; Stein, B. Cancer Res. 2002, 62, 1439.

[14] McKie, J. A.; Bhagwat, S. S.; Brady, H.; Doubleday, M.; Gayo, L.; Hickman, M.; Jalluri, R. K.; Khammungkhune, S.; Kois, A.; Mortensen, D.; Richard, N.; Sapienza, J.; Shevlin, G.; Stein, B.; Sutherland, M. Bioorg. Med. Chem. Lett. 2004, 14, 3407.

[15] Wang, C. N.; Wu, C. Y.; Zhu, J. Q.; Miller, R.; Wang, Y. M. J. Med. Chem. 2011, 54, 2331.

[16] Roussaki, M.; Kontogiorgis, C. A.; Hadjipavlou-Litina, D.; Hamilakis, S.; Detsi, A. Bioorg. Med. Chem. Lett. 2010, 20, 3889.

[17] Du, X. F.; Jiang, C. Z.; Wu, C. F.; Won, E. K.; Choung, S. Y. Arch. Pharm. Res. 2008, 31, 1153.

[18] Weng, F.; Wang, C. M.; Xu, B. Tetrahedron Lett. 2010, 51, 2593.

[19] Chen, H.; Gao, F.; Yin, Q. M.; Li, C. X.; Li, N.; Meng, M.; Li, X. L. Chin. J. Org. Chem. 2012, 32, 943 (in Chinese). (陈华, 高芳, 殷庆梅, 李春晓, 李娜, 孟明, 李小六, 有机化学, 2012, 32, 943.) 